\title{
Method of Laser Cross-correlation Optical Spectroscopy for Investigation of Dispersion Medium
}

\section{Z.A.Zabalueva, E.K. Nepomnyashchaya, E.N. Velichko, and E.T. Aksenov}

Peter the Great St. Petersburg Polytechnic University, Polytechnic Str. 29, St. Petersburg, 195251

\section{Abstract}

In the paper, we suggest one of the possible schemes of a cross-correlation optical spectroscopicdevice for investigation of turbid colloidal solutions and suspensions. This devise is designed fordeterminationof the hydrodynamic radii of nanoparticles. The cross-correlation function is constructed for two signals of light intensity scattered on a cuvette with the investigated dispersion medium. The construction of crosscorrelation optical spectroscopicdevice makes it possible to detect single scattered

Corresponding Author:

Z.A. Zabalueva

zoya199306@gmail.com

Received: 28 January 2018

Accepted: 15 March 2018

Published: 25 April 2018

Publishing services provided by Knowledge

(c) Z.A. Zabalueva et al. This article is distributed under the terms of the Creative Commons

Attribution License, which permits unrestricted use and redistribution provided that the original author and source are credited.

Selection and Peer-review under the responsibility of the PhIO Conference Committee. light by suppressing multiple scattering. We carried out an analysis of literature containing theory of light scattering and results of applying cross-correlation method for investigation of solutions and suspensions. Based on the analytical review, the scheme of cross-correlation optical spectroscopicdevice was developed and constructed.

Keywords: specklefield, cross-correlation, optical spectroscopy, size distribution, dispersion medium

\section{Introduction}

Inalmost every study of colloidal solutions or suspensions, there is a need to determine sizes of the particles in the suspension [1]. Not last place from all the physical methods, which are available today,belongs to optical methods. It is possible to distinguish a method of dynamic light scattering and a method of a cross-correlation optical spectroscopy. The first one was widely adopted due to accuracy and possibilities to investigate size distributions of most kinds of polydisperse particles [2]. It is widely used in medicine and pharmacology [3-5].However, the necessary objective is that the studied liquids must be diluted enough to hold the single scattering condition $[6,7]$. It seriously confines the dynamic light scattering application in many other areas such as geology, ecology and composite fluid studies. 
The method of a cross-correlation is quiet similar to the method of dynamic light scattering and allows measurements of size distributions in polydisperse liquids.In addition, it allows estimating not only the sizes of nanoparticles, but also other parameters, such as motion speeds, forms and internal diffusion coefficients without usage of polarizers or angle-dependency measurements [8-9]. It is also possible to study highly concentrated suspensions that significantly expands the area of cross-correlation optical spectroscopy applications.

Whereas all benefits, the cross-correlation optical spectroscopy is not still widely used because of the high complexity of experimental data processing, especially in comparison with dynamic light scattering method [9]. In this work, the main scope included the development of a scheme of cross-correlation optical spectroscopic device and a method of mathematical processing of experimental data aimed at determination of sizes distribution of nanoparticles in concentrated suspensions.

\section{Materials and methods}

The laser radiation scattered in a turbid medium undergoes accidental phase and amplitude shifts. As a result, chaotically located luminous spots called speckles can be observed [10]. Their set in the plane of observation is called a speckle field. Chaotic Brownian motion of nanoparticles in colloidal suspension leads to microscopic fluctuations of their local concentration that causes local fluctuations of the refraction index. The speckle field created by light scattered on this kind of environment changes in time and in space. The sizes of speckles, their form and time of intensity fluctuations depend on parameters of particles in the studied solution [11].For example, time of the speckle intensity fluctuation is connected to the speed of particles as

$$
\tau \approx \frac{a}{v}
$$

where $\tau$-time of the speckle intensity fluctuation, $v$-speed of particles, $a$-averaged size of speckles, which is equal to [11]

$$
a=\frac{\lambda z}{d}
$$

In this equation $\lambda$-wavelength of the scattered radiation, $z$-distance between the plane of dispersion and the plane of observation, $d$-size of illuminated area of a sample. 
By measuring the scattered light intensity fluctuations, in case of the assumption of spherical form of scattering nanoparticles, it is also possible to estimate their sizes and forms by using the Stokes-Einstein's equation [12]

$$
D=\frac{k_{B} T}{6 \pi \eta r} .
$$

Here, Bis the diffusion coefficient of nanoparticles, $\eta$ is the viscosity of the medium, $\mathrm{k}_{b}$ is the Boltzmann constant, $\mathrm{T}$ is the temperature, and $\mathrm{R}$ is the radius of nanoparticle.

To calculate the diffusion coefficient some spectral and correlation methods can be used. For instance, the method of the cross-correlation optical spectroscopy is based on the analysis of time and spectral characteristics of the scattered light. The intensity of the speckle field, created by moving particles, is measured in two different points, in which the scattered light is not coherent. The time dependent cross-correlation function is calculated as

$$
G_{12}(\tau)=\left\langle u_{1}(t+\tau) u_{2}(t)\right\rangle=\left\langle u_{2}(t+\tau) u_{1}(t)\right\rangle,
$$

where $u_{1}$ and $u_{2}$ time dependent intensity of the speckle field registered on two different angels, and triangular brackets mean averaging on time t. This function issymmetric, so we can consider only positive T.

In paper [11] it is said that for observation of temporal oscillations of a scattered light the differential scheme with two illuminating beamscan be used. The main benefit of such scheme consists in that the form of cross-correlation does not depend on the direction of observation, so the results of an experiment will not depend on the measurement angle.Deposits to cross-correlation function will be made only bysinglescattered light and by particles in the crossing of two dispersion volumes. No other fluctuations of scattered light intensity will contribute to cross-correlation function [11].

In the real experiment the cross-correlation function calculated for nanoparticles in free Brownian motion can be approximated as

$$
g_{12}(\tau)=A \exp \left[-2\left(K_{1} \tau-\frac{K_{2}}{2} \tau^{2}\right)\right]+1,
$$

where coefficient $A$ and cumulants $K_{1}$ and $K_{2}$ can be variable [9]. For polydisperse environments it is possible to use similar approximation of cross-correlation function

$$
g_{12}(\tau)=\int_{0}^{+\infty} \int_{0}^{+\infty} F\left(K_{1}, K_{2}\right) \exp \left[-2\left(K_{1} \tau-\frac{K_{2}}{2} \tau^{2}\right)\right] d K_{1} d K_{2}+1 .
$$

In this case $F\left(K_{1}, K_{2}\right), K_{1}, K_{2}$ can be variable too. The first cumulant $K_{1}$ is connected with diffusion coefficient by equation

$$
K_{1}=D q^{2},
$$


where $q$ is the length of the scattering vector

$$
q=\frac{4 \pi n}{\lambda_{0}} \sin \frac{\theta}{2}
$$

$\mathrm{n}$ is the refractive index of the medium, $\lambda_{0}$ is the wavelength, and $\theta$ is the angle of scattering.Using this equations and the Stokes-Einstein's equation one can calculate the size distribution of nanoparticles in studied medium.

It should be noted that there is a problem of approximation parameters values selection while finding of a smooth curve $\mathrm{F}\left(\mathrm{K}_{1}, \mathrm{~K}_{2}\right)$, which is stable to small changes of parameters $\mathrm{K}_{1}$ and $\mathrm{K}_{2}$. It means that it is necessary to select a processing method carefully. From our point of view the method of Tikhonov regularization allow finding of the solution which will be well agreed with a real data and is the most suitable for this kind of experiment [13].

\section{Results}

As was mentioned, the differential scheme with two illuminating beams has many benefits, so in this work it was chosen for the base of developed device. The designed schemeis presented in Figure 1. To create two equal illuminating beams the beam splitter and mirror was used.These beams are focused in the volume of cuvette with studied liquid suspension of nanoparticles. The scattered intensity is registered by square photodetectors.

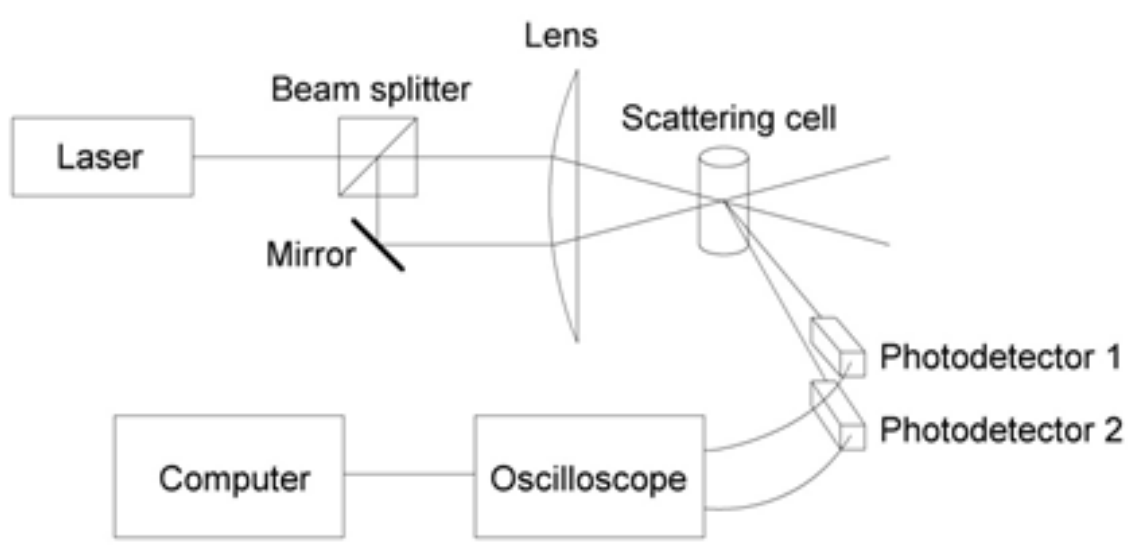

Figure 1: Scheme of the developed cross-correlation optical spectrometer.

In different directions, the integral intensity of scattered light is different. The direction, in which the intensity of light will be maximum, will be defined by wavelength of a laser radiation and the sizes of nanoparticles. Therefore, the possibility of turn and relocation of photo-sensor elements is provided in our device. 
From photodetectors signals arrive on a digital oscilloscope digitize signals and transfer them to the computer for further processing. We register two signals during several minutes, and then on the computer we break them into a number of smaller signals with identical length and calculate the cross-correlation function using each pair of them. The final cross-correlation functionis received by averaging.

To calculate size distribution from the cross-correlation function the modification of original algorithm based on the Tikhonov regularization was used. The algorithm is described in our previous works.

\section{Conclusion}

In the work, we developed a two-beam differential scheme for investigation of turbid colloidal solutions and suspensions using the cross-correlation method. Based on this scheme, we constructed a measuring device. Due to two coherent beams, we exclude the dependence of the experimental results on the observation angle of the scattered light fluctuations. Movable photodetectors provide the possibility of detecting at the maximum power of scattered light. The type of approximation of the cross-correlation function and the method of calculating for the parameters of the approximation by Tikhonov's regularization method was considered.

The created cross-correlation optical spectroscopic device would allow rapid analysis of turbid samples such as human blood in medicine and veterinary medicine, water and bog soils in ecological monitoring, lacquer varnish, paints and many others.

\section{References}

[1] Kelly Rae Chi, "Sizing up Nanoparticles," The Scientist, vol. 24, no 4, pp. 69-73, 2010.

[2] S. J. Douglas, L. Illum, S. S. Davis, and J. Creufer, "Particle Size and Size Distribution of Poly(Butyl-2-Cyanoacrylate) Nanoparticles: I. Influence of Physicochemical factors," Journal of Colloid and Interface Science, vol. 101, no 1, pp. 149-158, 1984.

[3] Devashri Sahu, G. M. Kannan, R. Vijayaraghavan, T. Anand, and Farhath Khanum, "Nanosized Zine Oxide Induces Toxicity in Human Lung Cells," ISRN Toxicology, vol. 2013, no 316075, pp. 1-8, 2013.

[4] A. N. Baranov, I. M. Vlasova, and A. M. Saletskii, "Investigation of Serum-Albumin Aggregation," Journal of Applied Spectroscopy, vol. 71, no 2, pp. 222-226, 2004.

[5] A. N. Baranov, I. M. Vlasova, V. E. Mikrin, and A. M. Saletskii, "Laser Correlation Spectroscopy of the Processes of Serum Albumin Denaturation," Journal of Applied 
Spectroscopy, vol. 71, no 6, pp. 911-915, 2004.

[6] J. H. Churnside and H. T. Yura, "Velocity Measurement Using Laser Speckle Statistics," Applied Optics, vol. 20, no 20, pp. 3539-3541, 1981.

[7] B. Chu, D. Fang, and Y. Mao, "Instrumentation of Multi-Scaled Scattering of BioMacromolecular Solutions," International Journal of Molecular Sciences, vol. 16, no 5, Pp. 10016-10037, 2015.

[8] M. C. Wilson and J. D. Harvey, "Twin-Beam Laser Velocimeter for the Investigation of Spermatozoon Motility," Biophysical Journal, vol. 41, January 1983.

[9] William V. Meyer, David S. Cannell, Anthony E. Smart, Thomas W. Taylor, and Padetha Tin. "Multiple-Scattering Suppression by Cross Correlation," Applied Optics, vol. 36, no 30, pp. 7551-7558, 1997.

[10] C. Urban and P. Schurtenberger, "Characterization of Turbid Colloidal Suspensions Using Light Scattering Techniques Combined with Cross-Correlation Methods," Journal of Colloid and Interface Science, vol. 207, no 1, pp. 150-158, 1998.

[11] S. S. Ul'yanov "Speckle Dynamics and Doppler Effect," Soros Educational Journal, vol. 7, no 10, pp. 1-7, 2001.

[12] E. Nepomnyashchaya, E. Velichko, E. Aksenov, "Inverse problem of laser correlation spectroscopy for analysis of polydisperse solutions of nanoparticles," Journal of Physics: Conference Series, vol. 769, no 1, pp. 012025, 2016.

[13] E. Nepomnyashchaya, Z. Zabalueva, E. Velichko, and E. Aksenov, "Modifications of laser correlation spectrometer for investigation of biological fluids," EPJ Web of Conferences, vol. 161, pp. 02017, 2017. 